\title{
On the rules for aquatic locomotion
}

\author{
M. Saadat,,${ }^{1,2,{ }^{*}}$ F. E. Fish, ${ }^{3}$ A. G. Domel, ${ }^{4}$ V. Di Santo, ${ }^{1}$ G. V. Lauder, ${ }^{1}$ and H. Haj-Hariri ${ }^{2}$ \\ ${ }^{1}$ Department of Organismal and Evolutionary Biology, Harvard University, Cambridge, \\ Massachusetts 02138, USA \\ ${ }^{2}$ Department of Mechanical Engineering, University of South Carolina, Columbia, \\ South Carolina 29208, USA \\ ${ }^{3}$ Department of Biology, West Chester University, West Chester, Pennsylvania 19383, USA \\ ${ }^{4}$ School of Engineering and Applied Sciences, Harvard University, Cambridge, Massachusetts 02138, USA
}

(Received 22 December 2016; published 18 August 2017)

\begin{abstract}
We present unifying rules governing the efficient locomotion of swimming fish and marine mammals. Using scaling and dimensional analysis, supported by new experimental data, we show that efficient locomotion occurs when the values of the Strouhal (St) number $\mathrm{St}(=f A / U)$ and $A^{*}(=A / L)$, two nondimensional numbers that relate forward speed $U$, tail-beat amplitude $A$, tail-beat frequency $f$, and the length of the swimmer $L$ are bound to the tight ranges of $0.2-0.4$ and $0.1-0.3$, respectively. The tight range of $0.2-0.4$ for the St number has previously been associated with optimal thrust generation. We show that the St number alone is insufficient to achieve optimal aquatic locomotion, and an additional condition on $A^{*}$ is needed. More importantly, we show that when swimming at minimal power consumption, the Strouhal number of a cruising swimmer is predetermined solely by the shape and drag characteristics of the swimmer. We show that diverse species of fish and cetaceans cruise indeed with the St number and $A^{*}$ predicted by our theory. Our findings provide a physical explanation as to why fast aquatic swimmers cruise with a relatively constant tail-beat amplitude of approximately $20 \%$ of the body length, and their swimming speed is nearly proportional to their tail-beat frequency.
\end{abstract}

DOI: 10.1103/PhysRevFluids.2.083102

\section{INTRODUCTION}

Scaling parameters are of great importance in describing the dominant physics of locomotion. The Strouhal ( $\mathrm{St})$ number $\mathrm{St}(=f A / U)$ is one such parameter that has been used widely to characterize aquatic locomotion [1-15] when the inertial fluid effects are dominant (high swimming velocities or large body sizes). The Strouhal number relates swimming speed $U$ to the tail-beat frequency $f$ and tail-beat amplitude (tip-to-tip excursion of the tail) $A$. This number can be thought of as an indicator for the effectiveness of the flapping locomotion as it indicates the distance a swimmer advances per flap of its tail. The Strouhal number was first introduced to characterize vortex shedding behind bluff bodies $[16,17]$. Owing to the presence of the vortex wake left behind both self-propelled swimmers and bluff bodies, the St number has been adopted to characterize thrust and efficiency as well as the wake structure of oscillating appendages used for self-locomotion [3].

Swimming and flying species differ in morphology and flapping mechanisms, but it has been observed that they share a common aspect of locomotion characterized by a narrow range of the St number [2-4]. This range of St number has previously been associated with maximum propulsive efficiency of oscillating foils [3]. In a typical study to investigate the performance of oscillating foils, the foil is placed inside a water tunnel with a prescribed incoming flow speed, and thrust and input power are measured for various foil kinematics. For both two-dimensional foils and three-dimensional wings, thrust and input power are found to increase with the St number. The propulsive efficiency-defined as the ratio of thrust power to input power-however exhibits a

*Author to whom correspondence should be addressed: sms722@fas.harvard.edu 
general peak in a range of the St number which in some studies [2-8] coincides with the narrow range of the St number observed in nature, whereas in some other studies $[9,10]$ there is no such correspondence. This tight range has also been attributed to the optimal duration that a leading edge vortex stays attached to the propulsor, which then can produce maximum possible thrust [1].

Although some of the experiments with prescribed incoming flow speed suggest that the tight range of the St number is related to the effectiveness of the swimming, the implication that the St number is actively tuned to this range by the animal remains speculative. In these experiments, the St number is considered as an independently controllable input parameter since the flow speed is prescribed and controlled separately. However in the self-propelled swimming of animals, $U$ is a variable that depends on the independent input variables $f$ and $A$. As such, there is no assurance that the St number can be tuned to the above range by the swimmer; therefore, it is effectively a dependent parameter [11,12]. A further question then is if indeed the animal cannot choose its St number and yet, its St number is observed to be tuned to the optimal range, how does this tuning take place, and what aspects of the design of the animal are most responsible for achieving this range of the Strouhal number? More importantly, given that the St number is not the sole indicator of efficiency-since many combinations of frequency and amplitude can result in the same St number $[6,13]$ — what additional conditions need to be satisfied so that economical locomotion of aquatic swimmers is realized fully?

Using simple dimensional and scaling analyses supported by new experimental evidence, we show that when cruising at minimum input power, the St number cannot actively be set by the swimmer. In particular, given the balance of thrust and drag in self-propelled swimming, it is shown that the St number is a function of only the size and drag coefficient of the shape. The drag coefficient is independent from $U$ at high speeds (turbulent flow), which then makes the St number (which depends explicitly on the drag coefficient and not on $U$ ) become independent of $U$ even implicitly. The independence of the St number from $U$ (at high speeds) further emphasizes the lack of ability by the swimmer to set its St number.

In this paper we provide further insight into the underlying conditions for optimal fishlike aquatic locomotion. We identify and propose a constraint on $A^{*}(=A / L)$ in addition to the well-referenced constraint on the St number [1-6] to fully describe the optimal swimming gait for fast swimmers $\left[\left(\operatorname{Re} \geqslant 10^{4}-10^{5}\right)\right.$ where Re stands for the Reynolds number] [18]. In particular, we show that the input power coefficient (a metric for the energy expenditure of the swimming) for a swimmer employing flapping fishlike locomotion is minimized for all cruising speeds only when the swimmer constrains $A^{*}$ to the narrow range of $0.1-0.3$. A survey of kinematics for a wide range of species of fish and cetaceans (both our data as well as data from the literature) shows that the ranges of the St number and $A^{*}$ for fast swimmers indeed are constrained to $0.2-0.4$ and $0.1-0.3$, respectively. Our findings provide a physical explanation as to why fast aquatic swimmers cruise with relatively constant tail-beat amplitude—at approximately $20 \%$ of the body length [14,15,19]—yielding a swimming speed which is nearly linearly correlated with their tail-beat frequency $[14,15]$.

\section{DIMENSIONAL AND SCALING ARGUMENTS}

We present a dimensional analysis that relates the swimming speed $U$ of a swimmer with fishlike flapping propulsion to the frequency $f$ and tip-to-tip amplitude $A$ of the tail beat as well as to the body length $L$ (Fig. 1). Note that kinematics of fish locomotion can be generally more complex. Here we aim to construct a model that captures the physics of fishlike aquatic locomotion using the minimum number of parameters.

We assume that most of the thrust force is produced by the lateral movement of the aft part of the swimmer (herein named the tail) and much of the resistive drag generated by its front part (herein named the body), which has considerably less movement than the posterior region of the body. Although the degree of separation of thrust and drag remains an open question [20], this assumption seems reasonable as a first approximation given previous work on fish kinematics which has shown for a diversity of species (from eels to tuna) that the anterior region of the body 


\section{ON THE RULES FOR AQUATIC LOCOMOTION}

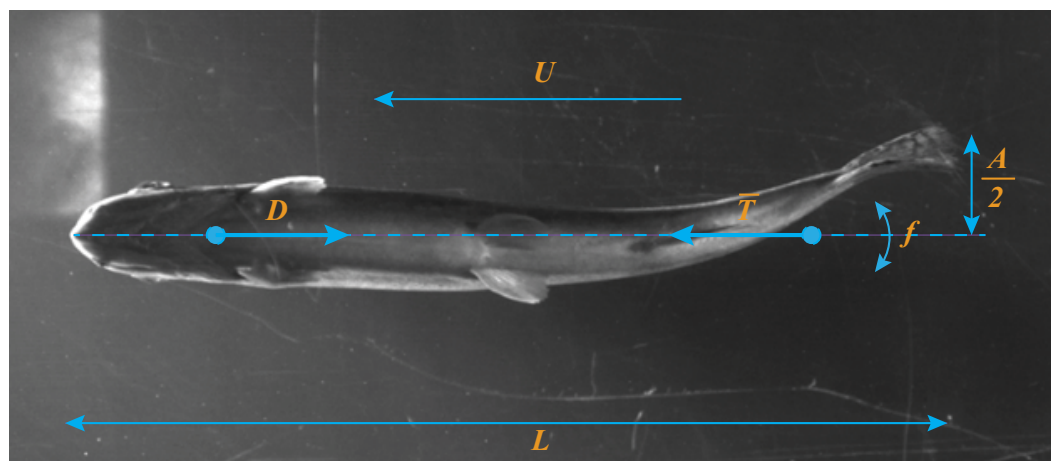

FIG. 1. A snapshot of a live freely swimming trout.

oscillates with much lower amplitude than the caudal (tail) area [21,22]. This has also been shown for swimming by cetaceans [23]. We further focus on the mean translation of the swimmer, constrained to the longitudinal direction, ignoring the translational and rotational oscillatory movements that are caused by the oscillation of the tail. This assumption is a starting point and is reasonable for fish swimming during rectilinear locomotion [24,25]. Indeed, a similar assumption has been invoked in a control-theoretic study [12] where the optimality of a three-linked swimmer moving in one dimension was studied. Additionally, we assume that the swimmer is slender, i.e., its body width is much smaller than its length.

For a freely swimming swimmer, the cycle averaged thrust force $\bar{T}$, produced by its tail is balanced by the resistive drag $\bar{D}$, mainly from its body,

$$
C_{T} S_{T}=C_{D} S_{D}
$$

where $C_{D}$ and $C_{T}$ are drag and thrust coefficients associated with the body and the tail, respectively, and defined as $C_{(\bar{T}, \bar{D})}=2(\bar{T}, \bar{D}) / \rho U^{2} S_{(T, D)}$ where $\rho$ is the density of the fluid and $S_{T}$ and $S_{D}$ are the lateral planform areas of the tail and the body.

The model we employ is a freely swimming swimmer that controls its speed through only two degrees of freedom, namely, tail-beat frequency and amplitude. Speed $U$ is an outcome of the effort; it is a function $\left(\phi_{1}\right)$ of frequency and amplitude of the tail beat, geometry, thrust characteristics of the tail, as well as geometry and drag characteristics of the body,

$$
U=\phi_{1}\left(f, A, L, C_{D}, C_{T}\right) .
$$

$C_{D}$ and $C_{T}$ contain information about the body and tail geometries, respectively. Furthermore, $C_{D}$ and $C_{T}$ are functions of the Reynolds number of the flow. Equation (1) is used to eliminate the explicit dependence of Eq. (2) on $C_{T}$. Nondimensionalizing the revised Eq. (2) results in

$$
\frac{U}{f A}=\mathrm{St}^{-1}=\phi_{2}\left(\frac{S_{D}}{S_{T}}, \frac{A}{L}, C_{D}\right),
$$

where $\phi_{2}$ denotes the functional form of the dependence as yet to be determined. A similar dimensional analysis was performed in Ref. [26] where the swimming speed of fish was derived as a function of the body length, drag coefficient, and metabolic rate.

Equation (3) indicates that the motion of a cruising fishlike swimmer can be described fully using two nondimensional numbers, the St number and $A^{*}(=A / L)$, implying both the significance as well as the insufficiency of the St number to fully characterize aquatic locomotion. In particular, Eq. (3) shows that, when the swimmer maintains constant amplitude of the tail beat, then the St number becomes only a function of geometry and drag characteristics of the fish and cannot be controlled otherwise. The assumption of the constancy of $A^{*}$ is motivated originally by the observation that fish and cetaceans maintain fairly constant tail-beat amplitudes at $20 \%$ of their body length when 
cruising $[14,15,19]$. In the following section we show that there is indeed an energetic advantage for a swimmer at all speeds when the nondimensional flapping amplitude of the motion is constrained to the tight range of $0.1-0.3$.

To gain more insight into the dependence of the St number of a swimmer on its geometry and drag characteristics, a scaling argument is undertaken. The focus remains on motions with high flapping frequencies and low flapping amplitudes because they are more efficient (as will be shown below). For such high- $f$ and low- $A$ motions, added mass effects are the dominant contributors for the production of force. The mass of fluid displaced by the oscillating tail in each tail beat scales with $\rho S_{T} A$ whereas the acceleration of the tail scales with $f^{2} A$. As a result, it makes sense to scale cycle-averaged thrust as $\bar{T} \sim \rho S_{T}(f A)^{2}$.A thrust coefficient $\tilde{C}_{T}$ corresponding to this scaling is then defined

$$
\tilde{C}_{T}=\frac{\bar{T}}{\frac{1}{2} \rho S_{T}(f A)^{2}} \rightarrow \tilde{C}_{T}=\frac{1}{\mathrm{St}^{2}} C_{T} .
$$

Theodorsen's thin airfoil theory as is covered in Ref. [27] indicates that $\tilde{C}_{T}$ is constant in an ideal flow (i.e., at the limit of infinite Re numbers). Based on this theory, for a thin pitching foil, the value for the constant is $\tilde{C}_{T}=\pi^{3} / 16$. The relation of $C_{T}$ with the St number as predicted in Eq. (4) agrees with the experimental findings of Ref. [28] where they showed time-averaged thrust coefficients of foils undergoing heaving and pitching motions correlated well with the square of the St number for a wide range of flow conditions.

Substituting the new expression for $C_{T}$ into Eq. (1) results in

$$
\mathrm{St}=\left(\frac{S_{D}}{S_{T}}\right)^{1 / 2}\left(\frac{C_{D}}{\tilde{C}_{T}}\right)^{1 / 2} .
$$

Absence of $A / L$ from Eq. (5) indicates that in the limit of high frequency and low amplitude flapping motions, the explicit dependence of the St number on $A / L$ in Eq. (3) vanishes. Comparing Eq. (5) with Eq. (3) suggests the existence of a limiting value for $A / L$ (herein named $A_{c}^{*}$ ) above which the St number is no longer determined solely based on the shape of the swimmer but also depends on the kinematics of flapping. We provide experimental evidence that this swimming regime (motions with $A^{*}>A_{c}^{*}$ ) is not energetically favorable for swimmers with fishlike locomotion. In order to spend the least power to cruise at any chosen speed, a swimmer needs to maintain a constant amplitude at $A=A_{c}^{*} L$ wherein the St number becomes effectively independent of the specific gait and is determined solely by the shape and design of the swimmer. A gait is defined as a unique combination of directly controllable swimming parameters, i.e., $f$ and $A$.

Similar scaling arguments have been carried out in Ref. [29] to describe undulatory fish locomotion at cruise. They however do not take into account the effect of the tail-beat amplitude in deriving the underlying relations. In fact, the tail-beat amplitude is assumed $a$ priori to be constant solely based on observations of fish data [14] and without providing any physical explanation as to why this is the case. In the current paper, we show that a more complete description of the physics of the flapping fish locomotion requires that the tail-beat amplitude $A$ be retained (as $A^{*}=A / L$ ) and that it be controlled independently [Eq. (3)]. The optimum value of $A^{*}$ then is shown to be determined from the power efficiency of the swimmer.

It has been reported that the St number for fish varies with swimming speed $[15,21,30]$. Equation (5) provides an explanation as to how the St number varies with $U$ : Variation of the St number with $U$ simply is inherited from the variation of $C_{D}$ with $U$. To obtain a qualitative understanding of the St number versus the $U$ trend, it is sufficient to substitute the behavior for the drag coefficient of streamlined bodies [Fig. 2(b)] into Eq. (5). In the general case of fish locomotion, the total drag exerted on the body consists of skin friction and form drag as well as profile drag due to the motion of the body relative to the fluid $[31,32]$. Here, we assume that the body of the swimmer moves in the forward direction steadily with negligible translational or rotational accelerations and that the total drag of the body is dominated by skin friction drag at lower Reynolds numbers and by 
(a)

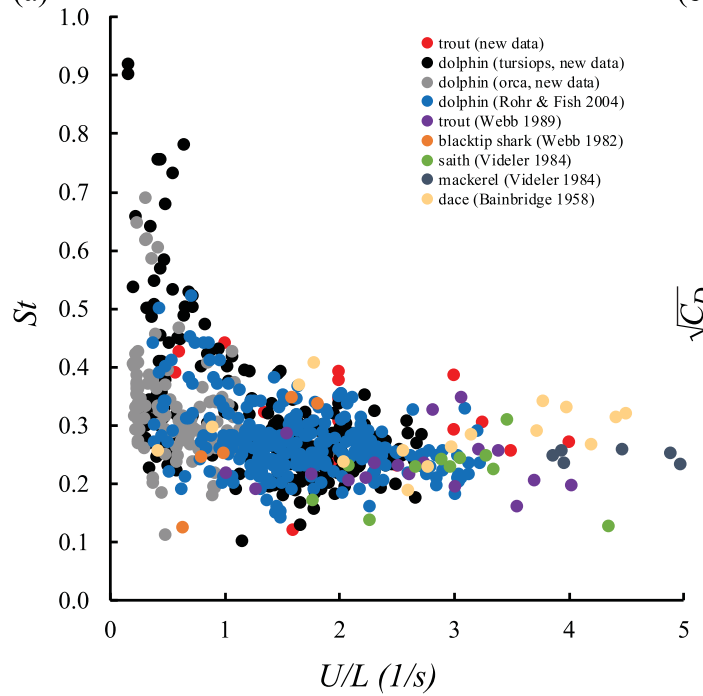

(b)

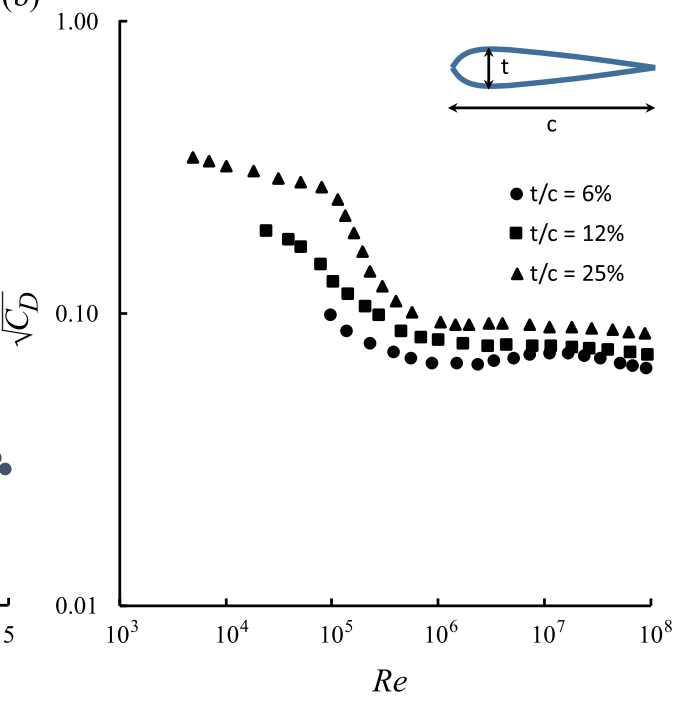

FIG. 2. (a) Variation of the Strouhal number with swimming speed per body length for trout (new data), cetaceans (new data), cetaceans [15] (various species of dolphin and killer whale), dace [14], mackerel [35], saithe [35], blacktip shark [36], and rainbow trout [37]. (b) (Square root of) The drag coefficient of streamlined bodies with thickness ratios of $6 \%, 12 \%$, and $25 \%$ as a function of the Reynolds number [38].

form drag at higher (turbulent) Re numbers [Fig. 2(b)]. This assumption is reasonable for bodies of most fish $[33,34]$.

According to Eq. (5), the St number is a decreasing function of $U$ with a pattern similar to the square root of the drag coefficient (of a streamlined body) with the Reynolds number: The St number drops rapidly with speed at low $U$ (where the skin friction drag dominates the total drag, i.e., viscous regime $D \sim U$ ) and eventually levels off at high enough $U$ (where the pressure drag dominates the total drag, i.e., inertial regime $D \sim U^{2}$ ). This trend of the St number versus $U$ predicted by Eq. (5) matches qualitatively with that observed among trout (new data), cetaceans (new data), cetaceans [15], dace [14], mackerel [35], saithe [35], blacktip shark [36], and rainbow trout [37] [Fig. 2(a)]. Figure 2(b) should not be taken as a quantitative match for Fig. 2(a). In order for Fig. 2(b) to be matched perfectly with Fig. 2(a), one would need to have access to the complete size and shape of the body as well as the speed-dependent drag coefficient for each swimmer. Such data were not available. The extent of the prediction of the theory in this paper is that the Strouhal number of a given animal has a dependence on speed that is inherited from the square root of the drag coefficient of the streamlined body.

Although the above dimensional and scaling analyses provide valuable insight into the physics of aquatic locomotion by identifying and clarifying the role of the governing nondimensional quantities, they do not contain information about the optimality of flapping fishlike swimming, which one may reasonably expect because of years of evolution that have contributed to energetically favorable locomotion for aquatic swimmers. To obtain information for the energetics of the motion, the detailed experimental analysis of models of flapping fishlike swimmers are undertaken next.

\section{EXPERIMENTAL METHODS}

A rectangular flexible foil of chord length $167 \mathrm{~mm}$ and span $68 \mathrm{~mm}$ was considered as a proxy for a fish performing undulatory locomotion. The foil was attached to an aluminum rod that was connected to a rotatory motor (Pittman, GM8724S009) through a force and torque transducer (ATI Nano-17 six-axis force and torque transducer, ATI, Inc.). The thickness of the foil was less than $3 \%$ 


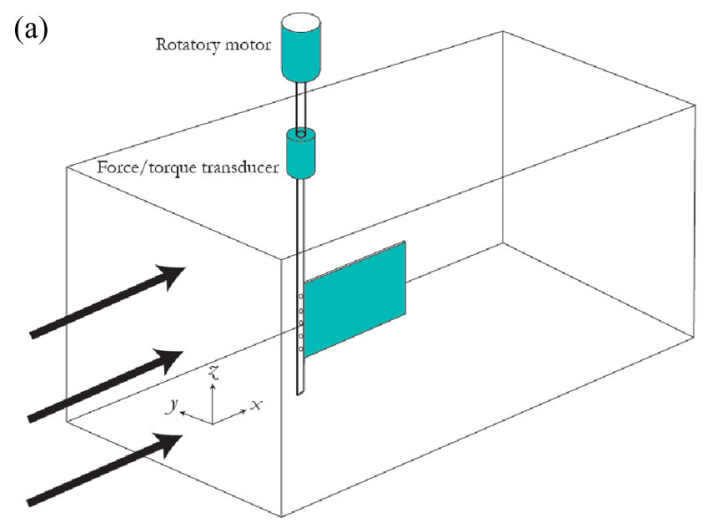

(b)

FIG. 3. Schematic of the robotic flapper to generate fishlike motion. (a) Perspective view and (b) top view.

of its chord length, constituting a slender thin fish model. The foil was oscillated at the leading edge with a pure sinusoidal pitch motion inside a recirculating flow tank at Lauder Laboratory, Harvard University, and the speed of the water in the flow tank was controlled by the data-acquisition program used to gather force and torque data and to drive the pitching motion. This apparatus has been used extensively in previous studies to analyze passively flexible models of fish propulsion [39-43]. Application of the pure pitch at the leading edge created body waves throughout the foil where the wave amplitude increased toward the trailing edge, closely resembling fish swimming. Pure pitch around the leading edge also minimized the creation of leading edge vortices which have been shown to greatly affect the forces on flapping foils [8]. A schematic of the device is shown in Fig. 3.

The controllable parameters of the system were frequency and amplitude of the pitch at the leading edge as well as the incoming flow speed of the flow tank. The tip-to-tip excursion of the trailing edge or amplitude $A$ was controlled through the input parameters. Parameters of interest are $f, A$, and $U$. The pitch amplitude acted merely as an intermediate parameter to generate the body waves, and its value is not reported here. To achieve the self-propelled condition, for a given prescribed flow speed, we searched through frequency-amplitude parameter space to find combinations of $f$ and $A$ that resulted in a zero time-averaged net $x$ force $\bar{F}_{x}$ on the foil. The $z$-torque $\tau$ around the leading edge then was measured for each combination of $f$ and $A$ to calculate the mechanical input power $P$ herein named the input power. The time-averaged input power $\bar{P}$ was calculated by integrating $\tau \dot{\theta}$ over time, where $\dot{\theta}$ is the $z$-angular velocity at the leading edge of the foil. $\bar{F}_{x}$ and $\bar{P}$ were measured over as many cycles as possible in $10 \mathrm{~s}$ of data reading, following an initial wait time so that transient effects were suppressed. We repeated the experiments for three different flow speeds corresponding to $1.15,1.57$, and 2 body lengths per second. The corresponding flow Reynolds number ranged from $\operatorname{Re}=32500$ to 56300 , where $\operatorname{Re}=U L / v$ and $v$ denotes the kinematic viscosity of the fluid.

Kinematic data on swimming Brook trout (Salvelinus fontinalis) were obtained using Photron PCI-1024 high-speed cameras with $1024 \times 1024$ pixel resolution. The trout swam steadily in the center of a recirculating flow tank with a working section of $28 \times 28 \times 66 \mathrm{~cm}(W \times H \times L)$ used for previous studies of fish swimming kinematics (e.g., Refs. [44,45]). Flow speeds ranged from one to three body lengths per second, water temperature was $14{ }^{\circ} \mathrm{C}$, and video sequences were saved only when fish swam in the center of the tank. Both side and bottom views were recorded simultaneously at a frame rate of 500 frames per second (fps). Fish tail-beat amplitudes and frequencies were measured from the two camera videos and were calibrated and aligned using direct linear transformation in MATLAB (MathWorks, Natick, MA) with software from Ref. [46].

Additionally, the swimming motions of seven species of trained odontocete whales were recorded at Sea World in Orlando, FL, San Antonio, TX, San Diego, CA, and the National Aquarium in Baltimore, MD. The species examined were from the family Delphinidae, including 1 spotted dolphin (Stenella frontalis), 6 Pacific white-sided dolphins (Lagenorhynchus obliquidens), 
(a)

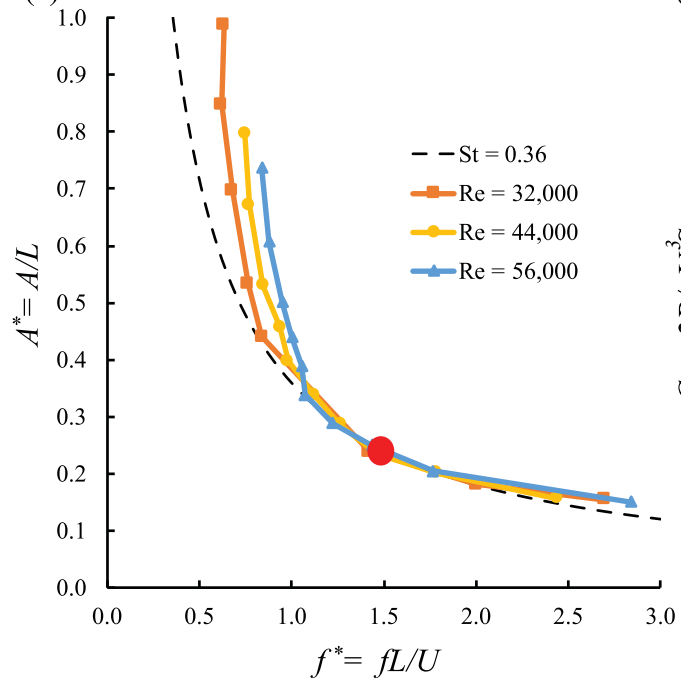

(b)

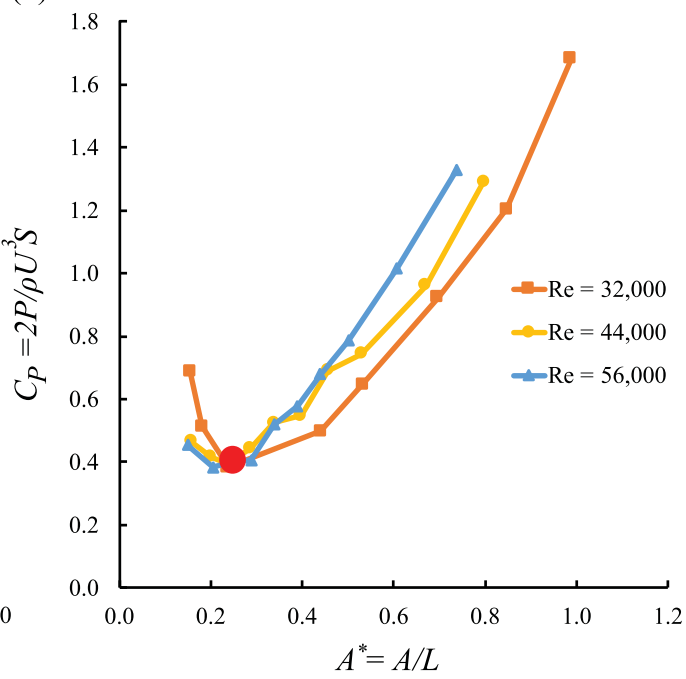

FIG. 4. Measured self-propelled speed and input power coefficients for the pitching foil under study: (a) Re number (dimensionless speed) contours (solid lines), and their corresponding St number contours (dashed line; $\mathrm{St}=f^{*} A^{*}$ ) and (b) power coefficient for each Re number contour as a function of nondimensional amplitude $A^{*} . A_{c}^{*}$ is denoted by a solid red disk.

30 bottlenose dolphins (Tursiops truncatus), 4 false killer whales (Pseudorca crassidens), 7 pilot whales (Globicephala melaena), and 15 killer whales (Orcinus orca), and the family Monodontidae, including 11 beluga whales (Delphinapterus leucas). Some of the data were reported previously [15]. The body length of each animal was measured on site and used as a scale for each video sequence. Experiments were performed in large elliptical pools with maximum lengths of 27.4-48.8 m. The front curved portions of each pool were constructed of 1.7-2.1-m-wide Plexiglas panels, allowing for an unobstructed view of the animals as they swam. A water depth of 1.4-2.1 m was visible through the panels. The depth of the pools was 7.3-11.0 m. The animals normally swam about $0.5-1.0 \mathrm{~m}$ below the surface of the water and $1 \mathrm{~m}$ from the pool walls. The animals were trained to swim upon command at different speeds around the circumference of the pool.

The swimming of the individual whales was video recorded with camcorders (Sony CCD-TR81, Panasonic DV-510, and Sony HDR-SR11) at a rate of $30 \mathrm{fps}$. Sequential body and fluke positions were digitized using the Peak Motus video analysis system (version 4.3.1, Peak Performance Technologies, Englewood, CO) or Proanalyst Professional (version 1.5.4.8, Xcitex, Cambridge, MA). Kinematic data from video records to calculate the Strouhal number included mean swimming speed $(U, \mathrm{~m} / \mathrm{s})$, fluke oscillation frequency $(f, \mathrm{~Hz})$ and peak-to-peak fluke amplitude $(A, \mathrm{~m}) . A$ is defined as the maximum vertical displacement of the trailing edge of the flukes. Only video sequences in which the animals appeared to be swimming horizontally and at a constant speed were used.

\section{EXPERIMENTAL RESULTS}

\section{A. Gait independence and shape dependence of the Strouhal number of a fishlike swimmer}

Figure 4 presents the measured kinematics of a self-propelled foil ( $\bar{F}_{x}=0$ for all cases) on the plane of nondimensional amplitude $A^{*}=A / L$ versus nondimensional frequency $f^{*}=f L / U$. The definition for $f^{*}$ is similar to reduced frequency $k$, used in some previous studies $[1,6,8]$ none of which addressed free swimming. Note that, although frequency $f$ is an independently controllable variable, its nondimensional form, i.e., $f^{*}=f L / U$ is a dependent parameter in free swimming because as $f$ changes, so does $U$, resulting in a behavior of $f^{*}$ which is not similar to $f$. The results 
were obtained for three different cruising speeds corresponding to Reynolds numbers of 32000 , 44000 , and 56000.

As seen in Fig. 4(a), for motions with $A^{*}$ less than a critical amplitude of $A_{c}^{*} \sim 0.2$, contours for all self-propelled speeds essentially coincide with one single contour of $\mathrm{St} \sim 0.36$, which falls in the observed range of 0.2-0.4 coincident with fish and cetaceans [4]. Coincidence of a single St number contour with multiple cruising speed contours below $A^{*} \sim 0.2$ shows that the St number of the motion is independent of the foil flapping kinematics so long as $A \leqslant A_{c}^{*} L$. For such motions, the $\mathrm{St}$ number is determined solely by the geometry of the swimmer as was suggested by the dimensional and scaling analyses and is independent of the specific gait ( $f$ and $A$ ).

Similar to the St number, $f^{*}$ is also a dependent variable for flapping fishlike swimmers in cruise. Its value cannot be set by the swimmer readily because the speed $U$ varies along with the frequency $f$. Therefore, the only way that a swimmer can move along the cruise speed lines in Fig. 4(a) is by altering the nondimensional amplitude of the motion $A^{*}$; the constancy of the St number fixes the value of $f^{*}$ as a constant too because $\operatorname{St}\left(=f^{*} A^{*}\right)$. As a result, the swimming speed of the swimmer remains proportional to its tail-beat frequency when the flapping amplitude is kept fixed, i.e., $U=\left(L / f^{*}\right) f$ since $L / f^{*}$ is effectively a constant for a flapping fishlike swimmer. This result explains the observation $[14,15]$ that the swimming speed of fish and cetaceans is nearly proportional to their tail-beat frequency. Finally, for motions with $A>A_{c}^{*} L$, the independence of the St number from the flapping gait no longer holds. The physical mechanism behind the deviation of constant speed lines from the St number contour on the $f^{*}-A^{*}$ plane remains an open topic for future investigation. The shedding of leading-edge vortices may be an important factor $[1,6]$.

\section{B. Minimum power gait}

Although the gaits for self-propulsion share a common St number (for $A^{*} \leqslant A_{c}^{*}$ ), they do vary in terms of input power as shown in Fig. 4(b). The input power coefficient $C_{P}=2 P / \rho U^{3} S$ is found to be minimum at the point where the isospeed contours begin to deviate from the cruising St number contour (St $\sim 0.36$ ) on the $f^{*}-A^{*}$ plane, i.e., at $A^{*}=A_{c}^{*}$ in Fig. 4(a). $S$ is the undeformed planform area of the foil. The value of $A_{c}^{*}$ was found to be $A_{c}^{*} \sim 0.2$. The current definition of $C_{P}$ has been used previously by Ref. [47] as one of their performance metrics in the shape-gait optimization of aquatic undulatory locomotion in cruise.

Reviewing the definition of $\mathrm{St}=f A / U, C_{P}$ can be rewritten as $C_{P}=2 P / \rho U^{3} S=$ $2 P \mathrm{St}^{2} / \rho(f A)^{2} S U$. Given that the cycle-averaged thrust of a cruising swimmer $\bar{T}$ scales with $\rho(f A)^{2} S, C_{P}$ can be approximated as $C_{P} \sim \mathrm{St}^{2} P / \bar{T} U=\mathrm{St}^{2} / \eta$, where $\eta$ is the Froude efficiency, defined as $\eta=\bar{T} U / P$. Finally, given that the $\mathrm{St}$ number of the swimmer remains fairly constant at high enough swimming speeds, $C_{P}$ then becomes equivalent to the inverse of efficiency $C_{P} \sim 1 / \eta$. In that respect, $C_{P}$ can be thought of as a measure for effectiveness of swimming at the cruise condition. When plotted in terms of $C_{P}$, the power data partially collapse towards a single curve for all speeds with the collapse being nearly perfect at $A^{*}=A_{c}^{*}$.

These experimental results suggest that a swimmer can minimize its input power for all (sufficiently high) cruising speeds by staying at the nondimensional point in the $\left(f^{*}, A^{*}\right)$ space corresponding to $A_{c}^{*}$ on its cruising (and shape and size-dependent) St number contour. Dimensionally, this means that the swimmer must maintain its tail-beat amplitude at the constant level of $A\left(=A_{c}^{*} L\right)$ and vary its speed by varying the frequency, i.e., $U=\left(L / f^{*}\right) f=\left(L A_{c}^{*} / \mathrm{St}\right) f=$ $f \times$ const. These predictions are in line with the kinematics observed for a diverse range of species of fish and cetaceans, such as trout, dace, saithe, mackerel, dolphin, and blacktip shark; these data are plotted in Fig. 5. For these species the peak-to-peak amplitude of the tail beat averages approximately $20 \%$ of the body length about $\left(A^{*} \sim 0.2\right)$, and the speed is nearly proportional to the frequency of the tail beat, i.e., $U / L \sim f / 1.5$ (where $f^{*} \sim 1.5$ ).

We emphasize that the assumption of the constant drag coefficient (i.e., high swimming $R e$ ) is essential for the independence of the (newly found) optimal condition for flapping fishlike locomotion ( $f^{*} \sim 1.5$ and $A^{*} \sim 0.2$ ) from the swimming speed. When the drag coefficient shows great variations 


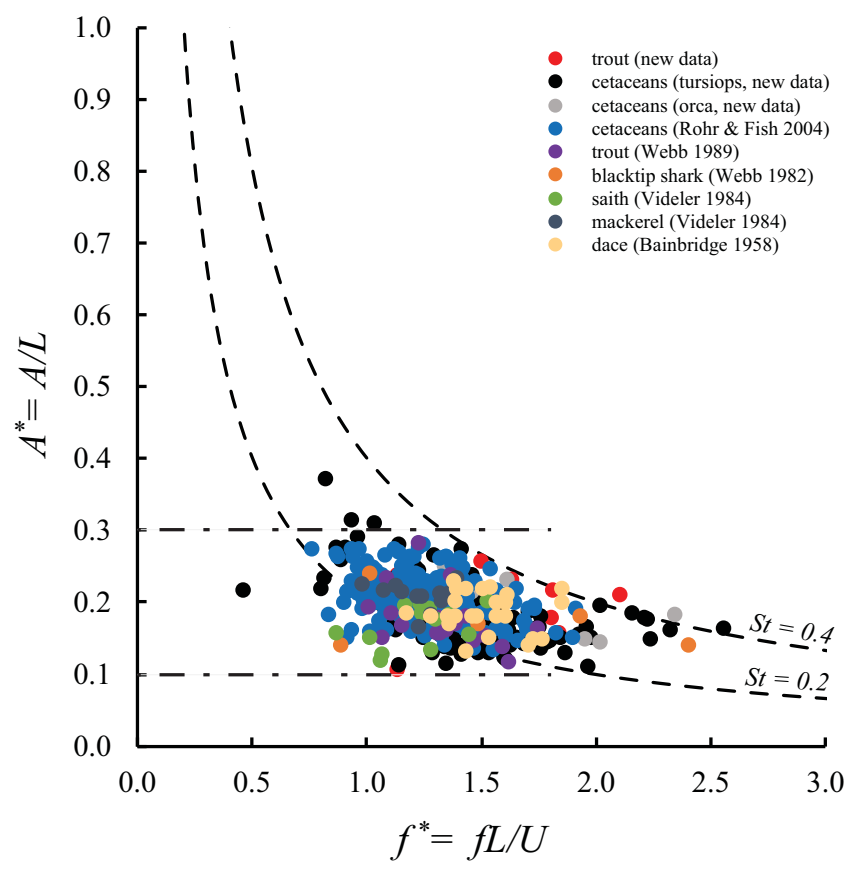

FIG. 5. Gait data for trout (new data), cetaceans (new data), cetaceans [15] (various species of dolphin and killer whale), dace [14], mackerel [35], saithe [35], blacktip shark [36], and rainbow trout [37] on the nondimensional plane of amplitude versus frequency. Only the data with sufficiently high speed [i.e., $U / L>$ $1(1 / \mathrm{s})]$ are reported to be consistent with the assumption of high-speed cruising. The dashed and dashed-dotted lines correspond to $\mathrm{St}=0.2$ and 0.4 and $A^{*}=0.1$ and 0.3 , respectively.

with speed (i.e., at small Re numbers), the numerical values for the optimal condition vary with speed. In the biological data that we obtained and processed, the St number seems to remain range bound $(\sim 0.2-0.4)$ when speed per body length of the swimmer is greater than unity (i.e., $U / L \geqslant 1)$.

Given the simplicity of the model utilized in the present paper, it is notable that the numerical values predicted for the critical tail-beat amplitude that maintains minimum energy expenditure of the locomotion for the swimmer matches the numerical values for the actual fish (Fig. 5 compared with Fig. 4). One possible reason for why the simple model seems to have both qualitative and quantitative bearings on the much more complex gaits in nature may be that the apparently more complex gaits also have very few controllable input parameters (degrees of freedom) and their apparent complexity merely is built passively into the actuation mechanisms (e.g., flexibility, etc.). An important element in any dimensional analysis is the actual number of independently controllable parameters (degrees of freedom). Thus, the number of independent variables appearing in dimensional and scaling analyses may not be too different between the simple model of this paper and the more complex fish locomotion in nature, leading to the good agreement.

\section{CONCLUSION}

It was shown that the kinematics of fish locomotion in a high-speed cruise is defined fully by two nondimensional parameters: (i) $\mathrm{St}=f A / U$, that relates the swimming speed $U$ to frequency $f$, and tip-to-tip amplitude of the tail $A$, and (ii) $A^{*}=A / L$, that relates the tip-to-tip tail-beat amplitude to the length $L$ of the swimmer. It was shown further that, when cruising at minimum input power, the St number cannot be set independently by the swimmer. Given the balance of thrust and drag forces when cruising, the St number is solely a function of the shape of the swimmer, i.e., drag coefficient 
and area. The St number does exhibit some variation with the speed. The trend by which the St number varies with swimming speed is related to the trend of variation of the drag coefficient of a streamlined body with the speed: They both drop quickly with speed at low speeds and level off (become constant) for high enough swimming speeds. The trend closely matches that exhibited by fish and cetaceans.

Additionally, detailed experimental measurements were carried out on the energetics of a selfpropelled fishlike flexible flapping foil as a surrogate for oscillatory fish locomotion. The results highlight the importance but also the insufficiency of the St number in describing efficient aquatic locomotion. It was shown that the input power coefficient (a measure of the energy expenditure of the swimming) is minimized when the length-specific flapping amplitude is constrained to a narrow range of $0.1<A^{*}<0.3$. Thus we identified a constraint on $A^{*}$ in addition to the previously found constraint on $\operatorname{St}(0.2-0.4)$ to fully describe the optimal swimming gait for fast swimmers. In agreement with our predictions, it is shown that diverse species of fish and cetaceans cruise with their St number and $A^{*}$ bound to the tight ranges of $0.2<\mathrm{St}<0.4$ and $0.1<A^{*}<0.3$.

The simple dimensional and scaling analyses in this paper, supported by experimental measurements, provide quantitative and qualitative explanations for the following observed behavior in animal locomotion: Fast aquatic swimmers cruise with relatively constant peak-to-peak tail-beat amplitude at approximately $20 \%$ of the body length [14,15,19], and their swimming speed is nearly proportional to their tail-beat frequency $[14,15]$.

\section{ACKNOWLEDGMENTS}

We thank Professor A. Smits, Professor H. Dong, and Professor H. Bart-smith for helpful discussions. H.H.-H. and M.S. gratefully acknowledge prior support provided by the Office of Naval Research under Program Director Dr. B. Brizzolara, MURI Grant No. N00014-08-1-0642. M.S. further acknowledges support from the Educational Foundation of the University of South Carolina. This research was further supported by the Office of Naval Research MURI Grant No. N00014-14-1-0533 (monitored by Dr. B. Brizzolara) to G.V.L. and F.E.F., and the Office of Naval Research Grant No. N00014-09-1-0352 (monitored by Dr. T. McKenna) to G.V.L.

M.S. and H.H.-H. developed the theory, performed the analysis, and wrote the paper. M.S. and G.V.L. conducted the experiments and interpreted the data. G.V.L. further helped with writing the paper. F.E.F. provided swimming kinematic data for fish and cetaceans. F.E.F. further helped with writing the paper. A.G.D. designed and fabricated the foil. V.D.S. provided swimming data for the trout.

[1] Z. J. Wang, Vortex shedding and frequency selection in flapping flight, J. Fluid Mech. 410, 323 (2000).

[2] M. S. Triantafyllou, G. S. Triantafyllou, and D. K. P. Yue, Hydrodynamics of fishlike swimming, Annu. Rev. Fluid Mech. 32, 33 (2000).

[3] M. S. Triantafyllou, G. S. Triantafyllou, and R. Gopalkrishnan, Wake mechanics for thrust generation in oscillating foils, Phys. Fluids A 3, 2835 (1991).

[4] G. K. Taylor, R. L. Nudds, and A. L. R. Thomas, Flying and swimming animals cruise at a Strouhal number tuned for high power efficiency, Nature (London) 425, 707 (2003).

[5] J. M. Anderson, K. Streitlien, D. S. Barrett, and M. S. Triantafyllou, Oscillating foils of high propulsive efficiency, J. Fluid Mech. 360, 41 (1998).

[6] G. C. Lewin and H. Haj-Hariri, Modelling thrust generation of a two-dimensional heaving airfoil in a viscous flow, J. Fluid Mech. 492, 339 (2003).

[7] R. P. Clark and A. J. Smits, Thrust production and wake structure of a batoid-inspired oscillating fin, J. Fluid Mech. 562, 415 (2006). 


\section{ON THE RULES FOR AQUATIC LOCOMOTION}

[8] P. A. Dewey, B. M. Boschitsch, K. W. Moored, H. A. Stone, and A. J. Smits, Scaling laws for the thrust production of flexible pitching panels, J. Fluid Mech. 732, 29 (2013).

[9] H. Dong, R. Mittal, and F. M. Najjar, Wake topology and hydrodynamic performance of low-aspect-ratio flapping foils, J. Fluid Mech. 566, 309 (2006).

[10] J. H. J. Buchholz and A. J. Smits, The wake structure and thrust performance of a rigid low-aspect-ratio pitching panel, J. Fluid Mech. 603, 331 (2008).

[11] M. Saadat and H. Haj-Hariri, 65th Annual Meeting of the APS Division of Fluid Dynamics, San Diego, 2012 (APS, San Diego, CA, 2012).

[12] S. Kohannim and T. Iwasaki, Analytical insights into optimality and resonance in fish swimming, J. R. Soc., Interface 11, 20131073 (2014).

[13] S. Alben, C. Witt, T. V. Baker, E. Anderson, and G. V. Lauder, Dynamics of freely swimming flexible foils, Phys. Fluids 24, 051901 (2012).

[14] R. Bainbridge, The speed of swimming of fish as related to size and to the frequency and amplitude of the tail beat, J. Exp. Biol. 35, 109 (1958).

[15] J. J. Rohr and F. E. Fish, Strouhal numbers and optimization of swimming by odontocete cetaceans, J. Exp. Biol. 207, 1633 (2004).

[16] A. Fage and F. C. Johansen, On the flow of air behind an inclined flat plate of infinite span, Proc. R. Soc. London, Ser. A 116, 170 (1927).

[17] A. Roshko, On the drag and shedding frequency of two-dimensional bluff bodies, National Advisory Committee for Aeronautics Report No. 3169, 1954.

[18] P. W. Webb, Form and function in fish swimming, Sci. Am. 251, 72 (1984).

[19] J. R. Hunter and J. R. Zweifel, Swimming speed, tail beat frequency tail beat amplitude, and size in jack mackerel, Trachurus symmetricus, and other fishes, Fish. Bull. 69, 253 (1971).

[20] W. W. Schultz and P. W. Webb, Power requirements of swimming: Do new methods resolve old questions? Integr. Comp. Biol. 42, 1018 (2002).

[21] G. V. Lauder and E. D. Tytell, in Fish Biomechanics, edited by R. E. Shadwick and G. V. Lauder, Fish Physiology Vol. 23 (Elsevier, Amsterdam, 2006), p. 425.

[22] G. V. Lauder and P. G. A. Madden, Advances in comparative physiology from high-speed imaging of animal and fluid motion, Annu. Rev. Physiol. 70, 143 (2008).

[23] F. E. Fish, K. T. Goetz, D. J. Rugh, and L. V. Brattstrom, Hydrodynamic patterns associated with echelon formation swimming by feeding bowhead whales (Balaena mysticetus), Mar. Mammal Sci. 29, E498 (2013).

[24] B. C. Jayne and G. V. Lauder, Speed effects on midline kinematics during steady undulatory swimming of largemouth bass, Micropterus salmoides, J. Exp. Biol. 198, 585 (1995).

[25] G. V. Lauder, in The Physiology of Fishes, 3rd ed., edited by D. H. Evans and J. B. Claiborne (Taylor \& Francis, 2006).

[26] T. Y. Wu, Fish swimming and bird/insect flight, Annu. Rev. Fluid Mech. 43, 25 (2011).

[27] J. Katz and A. Plotkin, Low-Speed Aerodynamics (Cambridge University Press, Cambridge, UK, 2001).

[28] T. C. W. Lau and R. M. Kelso, A scaling law for thrust generating unsteady hydrofoils, J. Fluids Struct. 65, 455 (2016).

[29] M. Gazzola, M. Argentina, and L. Mahadevan, Scaling macroscopic aquatic locomotion, Nat. Phys. 10, 758 (2014).

[30] I. Borazjani and F. Sotiropoulos, Numerical investigation of the hydrodynamics of carangiform swimming in the transitional and inertial flow regimes, J. Exp. Biol. 211, 1541 (2008).

[31] V. Raspa, S. Ramananarivo, B. Thiria, and R. Godoy-Diana, Vortex-induced drag and the role of aspect ratio in undulatory swimmers, Phys. Fluids 26, 041701 (2014).

[32] U. Ehrenstein and C. Eloy, Skin friction on a moving wall and its implications for swimming animals, J. Fluid Mech. 718, 321 (2013).

[33] E. J. Anderson, W. R. McGillis, and M. A. Grosenbaugh, The boundary layer of swimming fish, J. Exp. Biol. 204, 81 (2001).

[34] P. W. Webb, Hydrodynamics and energetics of fish propulsion, Bull. Fish. Res. Bd. Can. 190, 1 (1975). 


\section{SAADAT et al.}

[35] J. J. Videler and F. Hess, Fast continuous swimming of two pelagic predators, saithe (Pollachius virens) and mackerel (Scomber scombrus): A kinematic analysis, J. Exp. Biol. 109, 209 (1984).

[36] P. W. Webb and R. S. Keyes, Swimming kinematics of sharks, Fish. Bull. 80, 803 (1982).

[37] P. W. Webb, Steady swimming kinematics of tiger musky, an esociform accelerator, and rainbow-trout, a generalist cruiser, J. Exp. Biol. 138, 51 (1988).

[38] S. F. Hoerner, Fluid-Dynamic Drag: Practical Information on Aerodynamic Drag and Hydrodynamic Resistance (Hoerner Fluid Dynamics, Midland Park, NJ, 1965).

[39] K. L. Feilich and G. V. Lauder, Passive mechanical models of fish caudal fins: Effects of shape and stiffness on self-propulsion, Bioinspiration Biomimetrics 10, 036002 (2015).

[40] G. V. Lauder, E. J. Anderson, J. Tangorra, and P. G. A. Madden, Fish biorobotics: Kinematics and hydrodynamics of self-propulsion, J. Exp. Biol. 210, 2767 (2007).

[41] G. V. Lauder, B. Flammang, and S. Alben, Passive robotic models of propulsion by the bodies and caudal fins of fish, Integr. Comp. Biol. 52, 576 (2012).

[42] K. N. Lucas, P. J. M. Thornycroft, B. J. Gemmell, S. P. Colin, J. H. Costello, and G. V. Lauder, Effects of nonuniform stiffness on the swimming performance of a passively-flexing, fish-like foil model, Bioinspiration Biomimetrics 10, 056019 (2015).

[43] R. M. Shelton, P. J. M. Thornycroft, and G. V. Lauder, Undulatory locomotion of flexible foils as biomimetic models for understanding fish propulsion, J. Exp. Biol. 217, 2110 (2014).

[44] V. Di Santo, E. L. Blevins, and G. V. Lauder, Batoid locomotion: Effects of speed on pectoral fin deformation in the little skate, Leucoraja erinacea, J. Exp. Biol. 220, 705 (2017).

[45] E. M. Standen and G. V. Lauder, Hydrodynamic function of dorsal and anal fins in brook trout (Salvelinus fontinalis), J. Exp. Biol. 210, 325 (2007).

[46] T. L. Hedrick, Software techniques for two- and three-dimensional kinematic measurements of biological and biomimetic systems, Bioinspiration Biomimetrics 3, 034001 (2008).

[47] G. Tokić and D. K. P. Yue, Optimal shape and motion of undulatory swimming organisms, Proc. R. Soc. B 279, 3065 (2012). 